Pacific Journal of Mathematics

OPERATORS PRESERVING DISJOINTNESS ON
REARRANGEMENT INVARIANT SPACES 


\title{
OPERATORS PRESERVING DISJOINTNESS ON REARRANGEMENT INVARIANT SPACES
}

\author{
YURI A. ABRAMOVICH
}

\begin{abstract}
Let $X$ and $Y$ be two rearrangement invariant spaces on a measure space $(\Omega, \Sigma, \mu)$ with a finite, nonatomic measure $\mu$. We show that if there exists a non-zero order continuous disjointness preserving operator $T: X \rightarrow Y$, then $X \subseteq Y$. This result has many consequences. For example, if $T: L_{p}(\Omega, \Sigma, \mu) \rightarrow L_{q}(\Omega, \Sigma, \mu) \quad(0<p<q \leq \infty)$ preserves disjointness, then $T \equiv 0$.
\end{abstract}

1. Notation and preliminary facts. Recall that a (linear) operator $T: X \rightarrow Y$ between vector lattices is said to be a disjointness preserving operator if $\left|x_{1}\right| \wedge\left|x_{2}\right|=0$ in $X$ implies $\left|T x_{1}\right| \wedge\left|T x_{2}\right|=0$ in $Y$. All vector lattices are assumed to be Archimedean, and all operators on normed or linear metric spaces are assumed to be continuous.

Let $(\Omega, \Sigma, \mu)$ be a measure space with a finite $\sigma$-additive nonatomic measure and $S(\Omega, \Sigma, \mu)$ be the space of all (equivalence classes of) measurable real valued functions. Throughout the work we will use the representation of the space $S$ as the space $C_{\infty}(Q)$ of all continuous extended functions on the Stone space $Q$ of $S$. (See [10] for details.) We retain the same notation $\mu$ for the corresponding measure on $Q$, which is defined on the $\sigma$-algebra $\Sigma_{Q}$ consisting of all subsets of the form $(E \backslash N) \cup(N \backslash E)$, where $E$ is a clopen (closed and open) subset of $Q$ and $N$ is a first category subset of $Q$. It is well known that $\mu(D)=0$ if and only if $D$ is a nowhere dense subset of $Q$. (Any extremally disconnected space $Q$ with such a measure is sometimes called a hyperstonian space.) A subspace $X$ of $S(\Omega, \Sigma, \mu)$ is called a rearrangement invariant (r.i.) ideal if

(i) $X$ is an order ideal in $S$, and

(ii) If $x \in X, y \in S$, and $x$ and $y$ are equimeasurable, in symbols $x \sim y$, then $y \in X$.

If, in addition, $X$ is equipped with a Banach norm $\|\cdot\|$ such that

(iii) $x_{1}, x_{2} \in X$ and $\left|x_{1}\right| \leq\left|x_{2}\right| \Rightarrow\left\|x_{1}\right\| \leq\left\|x_{2}\right\|$, and

(iv) $x_{1}, x_{2} \in X$ and $x_{1} \sim x_{2} \Rightarrow\left\|x_{1}\right\|=\left\|x_{2}\right\|$,

then $X$ is called a r.i. Banach function space. We refer to [7] for the basic facts concerning r.i. ideals and Banach spaces. (Let us mention 
incidentally that up to an equivalent renorming (i), (ii), and (iii) imply (iv). See [1] or [7, p. 115].) All necessary information about Banach and vector lattices can be found in $[4,10]$.

2. The following theorem is the main result of this article.

THEOREM 1. If $X$ and $Y$ are r.i. ideals and $X \nsubseteq Y$, then every order continuous disjointness preserving operator $T: X \rightarrow Y$ is identically equal to zero, i.e., $T \equiv 0$.

We precede the proof of this theorem with several immediate corollaries.

Corollary 2. Let $X$ and $Y$ be two r.i. Banach function spaces and $X$ have order continuous norm. If $T: X \rightarrow Y$ is a nonzero disjointness preserving operator, then $X \subseteq Y$.

An alternative proof of this corollary can be obtained using Lemma 5.2 in [6].

COROLlaRY 3. There is no nontrivial disjointness preserving operator from $L_{p}(\Omega, \Sigma, \mu)$ into $L_{q}(\Omega, \Sigma, \mu)$ for $0<p<q \leq \infty$.

REMARK. In a special case of $L_{p}$-spaces $(1 \leq p \leq \infty)$, when $\Omega$ is an open subset of $R^{n}$ and $\mu$ is Lebesgue measure, this result was earlier obtained by a quite different method by M. Drahklin [5].

Corollary 4 (L. Potepun [9]). Order isomorphic r.i. ideals coincide. That is, if $X$ and $Y$ are order isomorphic r.i. ideals, then $X=Y$.

Proof. Let $T$ be an order isomorphism of $X$ onto $Y$. Obviously, $T$ and $T^{-1}$ are order continuous and, hence, by Theorem $1, X \subseteq Y$ and $Y \subseteq X$, i.e., $X=Y$. The original proof in [9] was much more difficult.

3. Three auxiliary lemmas. The space $Q$ and measure $\mu$ below are as defined above.

LemMa 5. Let $A$ be a nonvoid clopen subset of $Q$ and let $\varphi$ be a continuous open mapping from $A$ into $Q$. Put $B=\varphi(A)$. Then there exists a nonvoid clopen subset $B_{1}$ of $B$ and a constant $K>0$ such that for any measurable $D \subset B_{1}$

$$
K^{-1} \mu(D) \leq \mu\left(\varphi^{-1}(D)\right) \leq K \mu(D) .
$$


Proof. The set $B=\varphi(A)$ is evidently a clopen subset of $Q$. We introduce a new measure $\gamma$ on the $\sigma$-algebra $\Sigma_{Q}$ by letting $\gamma(D):=$ $\mu\left(\varphi^{-1}(D \cap B)\right), \quad\left(D \in \Sigma_{Q}\right)$. Obviously, $B$ is the support set of the measure $\gamma$. Let us verify that $\gamma$ is absolutely continuous with respect to $\mu$. Take an arbitrary measurable set $D$ with $\mu(D)=0$. Hence $D$ is nowhere dense in $Q$. Since $\varphi$ is open the set $\varphi^{-1}(D)$ $\left(=\varphi^{-1}(D \cap B)\right)$ is also nowhere dense and thus $\mu\left(\varphi^{-1}(D \cap B)\right)=0$. This proves that $\gamma$ is absolutely continuous with respect to $\mu$ and, consequently, by the Radon-Nikodym theorem there exists a nonnegative function $h \in L_{1}(\Omega, \Sigma, \mu)$ such that $\gamma(D)=\int_{D} h d \mu$ for each measurable set $D$. Take a nonvoid clopen subset $B_{1} \subset B$ and a constant $K>0$ so that $K^{-1} \leq h(q) \leq K$ for each $q \in B_{1}$. Clearly $B_{1}$ and $K$ satisfy the desired properties.

LemMa 6. Let $X$ and $Y$ be two r.i. ideals on a (finite nonatomic measure) space $(\Omega, \Sigma, \mu)$. If $X \nsubseteq Y$, then for each set $D \in \Sigma$ with $\mu(D)>0$ there is a function $x \in X$ such that its support $\operatorname{supp}(x) \subset D$ and $x \notin Y$. Moreover, $x$ can be chosen to be a step function.

The proof is straightforward and is omitted. We only mention that for infinite measures this lemma is false and it is the only place where the finiteness of the measure $\mu$ is essential (see 5.4 below).

LeMMA 7. Let $Y$ be a r.i. ideal and $\tilde{y}=\sum_{n=1}^{\infty} d_{n} \chi_{E_{n}} \in Y$ be a step function, where $\left\{E_{n}\right\} \quad(n=1,2, \ldots)$ is a sequence of pairwise disjoint measurable sets. Also, let $\left\{D_{n}\right\}$ be a second sequence of pairwise disjoint measurable sets such that $K^{-1} \leq \mu\left(D_{n}\right) / \mu\left(E_{n}\right) \leq K$ for some $K>0$. Then the step function $x=\sum_{n=1}^{\infty} d_{n} \chi_{D_{n}}$ likewise belongs to $Y$.

4. Proof of Theorem 1. Let $T: X \rightarrow Y$ be an order continuous disjointess preserving operator from $X$ into $Y$ and let $X \nsubseteq Y$. We must show that $T \equiv 0$. The gist of the proof lies in an application of the multiplicative representation of disjointness preserving operators obtained in [2].

By Theorem A in [2], the operator $T$ admits a global multiplicative representation, i.e., there exists a clopen set $E \subset Q$, a function $e \in$ $C_{\infty}(Q)$ and a continuous mapping $\varphi$ from $E$ into $Q$, such that for each $x \in X$ and each $q \in Q$

$$
(T x)(q)=e(q) x(\varphi(q)), \quad \text { if } q \in E, \quad \text { and } \quad(T x)(q)=0 \text { otherwise. }
$$


The order continuity of $T$ implies that the mapping $\varphi$ is open (see [2, Lemma 4.1] or [8, Prop. 8]). Without loss of generality we may assume that $T \geq 0$. If $T \not \equiv 0$, then the set $E$ is nonvoid and $E_{0}:=\{q \in E: 0<e(q)<\infty\}$ is a dense open subset of $E$. (It is possible that $E_{0}=E$.) Let us fix some constant $M>0$ such that the clopen set $A=\operatorname{cl}\left\{q \in E_{0}: M^{-1}<e(q)<M\right\}$ is nonvoid.

If we restrict the mapping $\varphi$ to $A$ and let $B=\varphi(A)$, then the continuous open mapping $\varphi: A \rightarrow B$ satisfies the conditions of Lemma 5. Therefore there exists a nonvoid clopen set $B_{1} \subset B$ and a constant $K>0$ such that $K^{-1} \leq \mu(D) / \mu\left(\varphi^{-1}(D) \cap A\right) \leq K$ for each measurable $D \subset B_{1}$. The condition $X \nsubseteq Y$ implies by Lemma 6 that there exists a step function $x=\sum_{n=1}^{\infty} d_{n} \chi_{D_{n}}$ such that $x \in X, x \notin Y, D_{n} \subset B_{1}$, and $D_{n} \cap D_{m}=0 \quad(n \neq m)$. Since $x \in X$, the function $y=T x \in Y$. Now let us express $y$ in terms of the multiplicative representation of $T$. We have

$$
\begin{aligned}
y & =T x=e(x \circ \varphi)=e(\cdot) x(\varphi(\cdot))=e(\cdot)\left(\sum_{n=1}^{\infty} d_{n} \chi_{D_{n}}\right)(\varphi(\cdot)) \\
& =e(\cdot) \sum_{n=1}^{\infty} d_{n} \chi_{D_{n}}(\varphi(\cdot))=e(\cdot) \sum_{n=1}^{\infty} d_{n} \chi_{\varphi^{-1}\left(D_{n}\right)}(\cdot) .
\end{aligned}
$$

Since $y \in Y$, we see that $y \chi_{A} \in Y$ and hence

$$
y \chi_{A}=e \sum_{n=1}^{\infty} d_{n} \chi_{\varphi^{-1}\left(D_{n}\right) \cap A} .
$$

As we know $e(q) \in\left[M^{-1}, M\right]$ for each $q \in A$ and therefore the function $\tilde{y}=\sum_{n=1}^{\infty} d_{n} \chi_{\varphi^{-1}\left(D_{n}\right) \cap A}$ belongs to $Y$ if and only if $y \chi_{A} \in Y$. Letting $E_{n}=\varphi^{-1}\left(D_{n}\right) \cap A$, we see that $\tilde{y}=\sum_{n=1}^{\infty} d_{n} \chi_{E_{n}} \in Y$ and $K^{-1} \leq \mu\left(D_{n}\right) / \mu\left(E_{n}\right) \leq K$. By Lemma 7 this implies that $x \in Y$, a contradiction, and the proof is finished.

5. Examples and comments. First, we show that the hypotheses of Theorem 1 cannot be weakened.

5.1. The condition $X \nsubseteq Y$ is essential, since if $X \subseteq Y$, then, the identity imbedding id: $X \rightarrow Y$ is a nonzero order continuous disjointness preserving operator.

5.2. Here we show that the assumption of order continuity of $T: X \rightarrow Y$ cannot be dropped. Indeed, let a r.i. space $X$ have a 
nonzero discrete functional $f$. Then for each $Y$ we can easily construct a nonzero disjointness preserving operator $T: X \rightarrow Y$. To this end take an arbitrary $y \in Y, y \neq 0$ and define $T x=f(x) y$. It is evident that $T \not \equiv 0$ and $T$ preserves disjointness. (A similar argument explains why we do not consider the case of atomic measure spaces. This case is of no interest since each discrete r.i. space always has a nonzero order continuous discrete functional.)

5.3. Recall that a norm $\|\cdot\|$ on a normed lattice $Z$ is said to be strictly monotone if $0 \leq z_{1}<z_{2}$ implies $\left\|z_{1}\right\|<\left\|z_{2}\right\|$.

Proposition 8. If $X$ and $Y$ are r.i. Banach function spaces with strictly monotone norms and $T$ is a positive isometry from $X$ into $Y$, then $X \subseteq Y$ (and $X=Y$ if $T$ is also onto).

Proof. It is easy to see (and this observation is due to A. S. Veksler) that each positive isometry preserves disjointness provided the norm in $Y$ is strictly monotone. Thus, Theorem 1 is applicable and hence $X \subseteq Y$. If $T$ is also onto, then, as is shown in [3, Thm. 1], $T$ is necessarily an order isomorphism, and now Corollary 4 yields the desired equality $X=Y$.

5.4. The case of infinite measure. Let us assume that $\mu(\Omega)=\infty$. It is a little bit surprising that Theorem 1 does not hold in this case. A simple example is as follows. Take $X=L^{2}(\mathbf{R})$ and $Y=L^{2}(\mathbf{R}) \cap$ $L^{1}(\mathbf{R})$. Clearly $X$ and $Y$ are r.i. Banach function spaces with order continuous norms, $X \nsubseteq Y$ but, nevertheless, there exist nonzero order continuous disjointness preserving operators from $X$ into $Y$. For example, $T_{1} x:=x \chi_{[a, b]}$ (where $a<b$ are arbitrary real numbers), or $T_{2} x(t):=x(t) /\left(t^{2}+1\right)$ are such operators. Nevertheless, the following version of Theorem 1 still holds.

COROLlARY 9. Let $\mu(\Omega)=\infty$. If there exists a nonzero order continuous disjointness preserving operator $T: X \rightarrow Y$, where $X$ and $Y$ are r.i. ideals, then for each set $D$ of finite measure the subspace $X_{D}=\{x \in X: \operatorname{supp}(x) \subset D\}$ belongs to $Y$.

Proof. Since $T \not \equiv 0$ and $T$ is order continuous there is $x_{1} \in X$ such that $y_{1}=T x_{1} \neq 0$ and $\mu\left(E_{1}\right)<\infty$ where $E_{1}=\operatorname{supp}(x)$. Choose a set $E_{2}$ of finite measure for which $y_{1} \chi_{E_{2}} \neq 0$. Now put $E=E_{1} \cup E_{2} \cup D$ and define $T_{E}$ by $T_{E} x=\chi_{E} T\left(x \chi_{E}\right)$. Obviously, $T_{E}$ 
is a nonzero order continuous disjointness preserving operator from the r.i. ideal $X_{E}$ into the r.i. ideal $Y_{E}$. By Theorem $1, X_{E} \subseteq Y_{E}$. In particular, $X_{D} \subset Y$.

We have treated the case of real spaces only, but the results remain true for complex spaces as well.

In conclusion the author would like to thank Drs. E. Arenson, A. Kitover and A. Mekler for many stimulating discussions, and the referee for his help and valuable suggestions.

\section{REFERENCES}

[1] Y. A. Abramovich, On rearrangement invariant spaces, Funktsional. Anal. i Prilozhen., 9 (1975), No. 1, 47-48 (Russian).

[2] _ Multiplicative representation of disjointness preserving operators, Proc. Netherl. Acad., A86 (1983), f. 3, 265-279.

[3] _ Isometries of the normed lattices, Optimizatsiya, 43 (1988), 74-80 (Russian).

[4] C. D. Aliprantis and O. Burkinshaw, Positive Operators, Academic Press, New York and London, 1985.

[5] M. E. Drahklin, Inner superposition operator in spaces of integrable functions, Izvestiya VUZov, Mathematics, 5 (1986), 18-24 (Russian).

[6] W. B. Johnson, B. Maurey, G. Schechtman, and L. Tzafriri, Symmetric structures in Banach spaces, Mem. Amer. Math. Soc., 217 (1979).

[7] J. Lindenstrauss and L. Tzafriri, Classical Banach Spaces II, Springer-Verlag, 1979.

[8] A. A. Mekler and N. F. Sokolovskaya, The Nakano subspaces of conditionally complete vector lattices, Functional Analysis, Ul'anovsk, (1982), 92-101 (Russian).

[9] L. I. Potepun, On isomorphisms of rearrangement invariant spaces, Sibirsk. Math. J., 12 (1971), No. 3, 623-629 (Russian).

[10] B. Z. Vulikh, Introduction to the theory of partially ordered spaces, Moscow, 1961 (Russian) (English Translation, Noordhoff, Groningen, 11068).

Received July 18, 1988 and in revised form January 19, 1990.

INDIANA UNIVERSITY-PURDUE UNIVERSITY AT INDIANAPOLIS

INDIANAPOLIS, IN 46205-2810 


\section{PACIFIC JOURNAL OF MATHEMATICS EDITORS}

\author{
V. S. VARADARAJAN \\ (Managing Editor) \\ University of California \\ Los Angeles, CA 90024-1555-05 \\ Herbert Clemens \\ University of Utah \\ Salt Lake City, UT 84112 \\ THOMAS ENRIGHT \\ University of California, San Diego \\ La Jolla, CA 92093
}

R. FINN

Stanford University

Stanford, CA 94305

HeRmann FlaschKa

University of Arizona

Tucson, AZ 85721

VAUghan F. R. Jones

University of California

Berkeley, CA 94720

SteVEN KeRCKHOFF

Stanford University

Stanford, CA 94305

\section{C. MOORE}

University of California

Berkeley, CA 94720

MARTIN SCHARLEMANN

University of California

Santa Barbara, CA 93106

HAROLD STARK

University of California, San Diego

La Jolla, CA 92093

\section{ASSOCIATE EDITORS \\ R. ARENS \\ E. F. BECKENBACH \\ B. H. NeUmanN \\ F. WoLF \\ (1904-1989) \\ K. Yoshida (1906-1982)}

\section{SUPPORTING INSTITUTIONS}

UNIVERSITY OF ARIZONA

UNIVERSITY OF BRITISH COLUMBIA

CALIFORNIA INSTITUTE OF TECHNOLOGY

UNIVERSITY OF CALIFORNIA

MONTANA STATE UNIVERSITY

UNIVERSITY OF NEVADA, RENO

NEW MEXICO STATE UNIVERSITY

OREGON STATE UNIVERSITY
UNIVERSITY OF OREGON

UNIVERSITY OF SOUTHERN CALIFORNIA

STANFORD UNIVERSITY

UNIVERSITY OF HAWAII

UNIVERSITY OF TOKYO

UNIVERSITY OF UTAH

WASHINGTON STATE UNIVERSITY

UNIVERSITY OF WASHINGTON

The Supporting Institutions listed above contribute to the cost of publication of this Journal, but they are not owners or publishers and have no responsibility for its content or policies.

Mathematical papers intended for publication in the Pacific Journal of Mathematics should be in typed form or offset-reproduced (not dittoed), double spaced with large margins. Please do not use built up fractions in the text of the manuscript. However, you may use them in the displayed equations. Underline Greek letters in red, German in green, and script in blue. The first paragraph must be capable of being used separately as a synopsis of the entire paper. In particular it should contain no bibliographic references. Please propose a heading for the odd numbered pages of less than 35 characters. Manuscripts, in triplicate, may be sent to any one of the editors. Please classify according to the 1991 Mathematics Subject Classification scheme which can be found in the December index volumes of Mathematical Reviews. Supply name and address of author to whom proofs should be sent. All other communications should be addressed to the managing editor, or Elaine Barth, University of California, Los Angeles, California 90024-1555-05.

There are page-charges associated with articles appearing in the Pacific Journal of Mathematics. These charges are expected to be paid by the author's University, Government Agency or Company. If the author or authors do not have access to such Institutional support these charges are waived. Single authors will receive 50 free reprints; joint authors will receive a total of 100 free reprints. Additional copies may be obtained at cost in multiples of 50 .

The Pacific Journal of Mathematics (ISSN 0030-8730) is published monthly except for July and August. Regular subscription rate: $\$ 190.00$ a year (10 issues). Special rate: $\$ 95.00$ a year to individual members of supporting institutions.

Subscriptions, orders for numbers issued in the last three calendar years, and changes of address should be sent to Pacific Journal of Mathematics, P.O. Box 969, Carmel Valley, CA 93924, U.S.A. Old back numbers obtainable from Kraus Periodicals Co., Route 100, Millwood, NY 10546.

The Pacific Journal of Mathematics at P.O. Box 969, Carmel Valley, CA 93924 (ISSN 0030-8730) is published monthly except for July and August. Second-class postage paid at Carmel Valley, California 93924, and additional mailing offices. Postmaster: send address changes to Pacific Journal of Mathématics, P.O. Box 969, Carmel Valley, CA 93924.

PUBLISHED BY PACIFIC JOURNAL OF MATHEMATICS, A NON-PROFIT CORPORATION Copyright (C) 1991 by Pacific Journal of Mathematics 


\section{Pacific Journal of Mathematics}

Vol. 148, No. $2 \quad$ April, 1991

Yuri A. Abramovich, Operators preserving disjointness on rearrangement

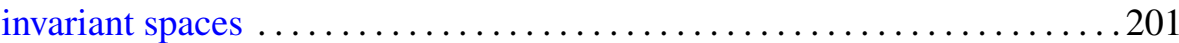

Andrew French Acker and Kirk Lancaster, Existence and geometry of a free boundary problem for the heat equation .................. 207

So-Chin Chen, Real analytic regularity of the Szegő projection on circular

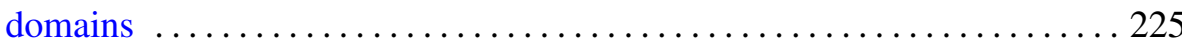

Chen-Lian Chuang, An independence property of central polynomials . . . 237

Peter Larkin Duren and M. Schiffer, Robin functions and energy

functionals of multiply connected domains $\ldots \ldots \ldots \ldots \ldots \ldots \ldots \ldots . \ldots 251$

Johan Henricus Bernardus Kemperman, Sets of uniqueness and systems

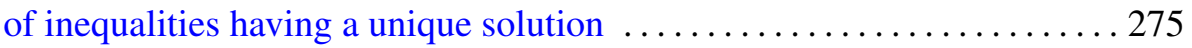

Ka-Lam Kueh, Fourier coefficients of nonholomorphic modular forms and sums of Kloosterman sums . .......................... 303

Gerard J. Murphy, Ordered groups and crossed products of $C^{*}$-algebras . . 319

You-Qiang Wang, The $p$-parts of Brauer character degrees in $p$-solvable

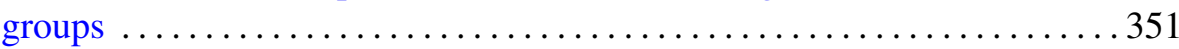

Hidenobu Yoshida, Harmonic majorization of a subharmonic function on a cone or on a cylinder ........................................ 369 